Dept. of Math./CMA University of Oslo $\begin{array}{lr}\text { PuRe Mathematics } & \text { No } 10 \\ \text { ISSN 0806-2439 } & \text { MaY } 2006\end{array}$

\title{
Optimal Portfolio, Partial Information and Malliavin Calculus
}

\author{
Giulia Di Nunno ${ }^{1}$ and Bernt $\varnothing_{k s e n d a l}{ }^{1,2}$
}

Revised, 15th July 2008.

\begin{abstract}
In a market driven by Lévy processes, we consider an optimal portfolio problem for a dealer who has access to some information in general smaller than the one generated by the market events. In this sense we refer to this dealer as having partial information. For this generally incomplete market and within a non-Markovian setting, we give a characterization for a portfolio maximizing the expected utility of the final wealth. Techniques of Malliavin calculus are used for the analysis.
\end{abstract}

Key words and phrases: stochastic control, partial information, optimal portfolio, utility maximization, Malliavin calculus.

AMS (2000) Classification: 93E20, 91B28, 60H07.

\section{Introduction}

During the last decade there have been many applications of Malliavin calculus to finance in the literature. So far the applications have essentially been within one of the following two areas:

\footnotetext{
${ }^{1}$ Centre of Mathematics for Applications (CMA) and Department of Mathematics, University of Oslo, P.O. Box 1053 Blindern, N-0316 Oslo, Norway.

${ }^{2}$ Norwegian School of Economics and Business Administration, Helleveien 30, N-5045 Bergen, Norway.

E-mail addresses: giulian@math.uio.no; oksendal@math.uio.no
} 
(i) Explicit formulae for the replicating portfolios or closest hedge of given claims essentially based on the Clark-Ocone formula (see e.g. [KO], [BDLØP])

(ii) Numerically tractable formulae for the computation of the "Greeks" in finance, for example the $\delta$-hedge (see e.g. [FLLLT]).

The purpose of this paper is to present a different type of financial application of Malliavin calculus, namely in the problem of finding an optimal portfolio. More precisely, we are given a utility function and we seek a portfolio (within an admissible class of portfolios) which maximizes the utility of the corresponding terminal wealth. This is a stochastic control problem. Traditionally there have been two approaches to this type of problem:

(a) A dynamic programming or stochastic maximum principle approach. These approaches require that the system is Markovian. See e.g. [YZ], and references therein, for more information about these methods.

(b) The martingale approach or duality approach. This is an efficient method if the financial market equations have a special "multiplicative" form (see (3.1)-(3.2)) and the market is complete. An advantage of the method is that the system need not be Markovian. However, in the incomplete market case the duality method transforms the original problem into a dual problem usually of equal difficulty. We refer to e.g. [KS], and references therein, for a presentation of the martingale method.

There are, however, many situations where the problem of finding the optimal portfolio cannot be approached by any of the two methods (a) and (b) above. For example, if the information $\mathcal{E}_{t}$ available to the agent at time $t \geq 0$ is strictly less than the information $\mathcal{F}_{t}$ that can be obtained by perfect observations of the processes driving the market prices up to time $t$, then the system may be neither Markovian nor complete. This is the case if, for example,

$$
\mathcal{E}_{t}=\mathcal{F}_{(t-\delta)^{+}}, \quad t \geq 0,
$$

where $\delta>0$ is a fixed information delay.

In this paper we will show that Malliavin calculus can be used to solve optimal portfolio problems also in such partial information cases.

We emphasize the distinction between the partial information control problem studied in this paper and the partial observation control problem, where the control is based on noisy observations of the state. The latter problem has been studied by many authors, but the methods and results of the partial observation problem do not apply to our situation. To the best of our knowledge, our paper is the first to investigate partial information optimal portfolio problems. Our approach uses a perturbation argument combined with Malliavin calculus. 
Our main result (Theorem 3.1) characterizes the existence of the optimal portfolio $\pi$ as solution of an explicit equation. We remark that this equation relates $\pi$ to the coefficients in the market equations and to the Malliavin derivatives of a certain function of the optimal terminal wealth $X_{\pi}(T)$.

\section{Some basic definitions and results}

\subsection{Lévy processes}

We first recall that a Lévy process $\eta=\eta(t)=\eta(t, \omega),(t, \omega) \in[0, \infty) \times \Omega$, on a complete probability space $(\Omega, \mathcal{F}, P)$ is a stochastically continuous process with stationary and independent increments. From now on we consider the version of $\eta$ which has càdlàg paths (i.e. right continuous paths with left sided limits) - see e.g. [S] for the existence of such a modification. We assume that $\eta(0)=0$. The (possible) jump of $\eta$ at time $t$ is defined by

$$
\Delta \eta(t)=\eta(t)-\eta\left(t^{-}\right) .
$$

The jump measure $N$ of $\eta$ is defined by

$$
N((a, b], U):=\sum_{t \in(a, b]} 1_{U}(\Delta \eta(t)),
$$

i.e. by the number of jumps of size $\Delta \eta(t) \in U$ for $t \in(a, b]$. Here $0 \leq a<b \leq \infty$ and $U \in \mathcal{B}\left(\mathbb{R}_{0}\right)$, i.e. $U$ is a Borel set with $\bar{U} \subset \mathbb{R}_{0}:=\mathbb{R} \backslash\{0\}$. The differential form of this random measure is denoted by $N(d t, d z)$. The Lévy measure of $\eta$ is defined by

$$
\nu(U):=E[N((0,1], U)], \quad U \in \mathcal{B}\left(\mathbb{R}_{0}\right)
$$

and the compensated jump measure (or compensated Poisson random measure) of $\eta$ is defined by

$$
\tilde{N}(d t, d z)=N(d t, d z)-\nu(d z) d t .
$$

In the sequel we assume that

$$
E\left[\eta^{2}(t)\right]<\infty, \quad t \geq 0 .
$$

The Lévy-Itô decomposition theorem states that if (2.1) holds, then there exist constants $a$ and $b$ such that

$$
\eta(t)=a t+b B(t)+\int_{0}^{t} \int_{\mathbb{R}_{0}} z \widetilde{N}(d s, d z), \quad t \geq 0,
$$


where $B$ is a standard Brownian motion independent of $N$. In view of this we see that it is natural to study processes which have the form

$$
X(t)=X(0)+\int_{0}^{t} \alpha(s) d s+\int_{0}^{t} \beta(s) d B(s)+\int_{0}^{t} \int_{\mathbb{R}_{0}} \gamma(s, z) \widetilde{N}(d s, d z),
$$

or, in differential form,

$$
d X(t)=\alpha(t) d t+\beta(t) d B(t)+\int_{\mathbb{R}_{0}} \gamma(t, z) \widetilde{N}(d t, d z) .
$$

The processes $\alpha=\alpha(t), \beta=\beta(t)$ and $\gamma=\gamma(t, z), t \geq 0, z \in \mathbb{R}_{0}$, are predictable with respect to the right continuous filtration $\mathbb{F}$ on $(\Omega, \mathcal{F}, P)$. Here $\mathbb{F}:=\left\{\mathcal{F}_{t}, t \geq 0\right\}$, is generated by $B$ and $N$ and $\mathcal{F}_{0}$ is augmented of all $P$-null events. Moreover it is assumed that

$$
E \int_{0}^{t}\left\{|\alpha(s)|+\beta^{2}(s)+\int_{\mathbb{R}_{0}} \gamma^{2}(s, z) \nu(d z)\right\} d s<\infty \quad P-\text { a.s. } \quad \text { for all } t \geq 0 .
$$

In particular, solutions of stochastic differential equations of the form

$$
d X(t)=\alpha(X(t)) d t+\beta(X(t)) d B(t)+\int_{\mathbb{R}_{0}} \gamma\left(X\left(t^{-}\right), z\right) \widetilde{N}(d t, d z)
$$

are called jump diffusions. We refer to $[\mathrm{A}]$ and $[\mathrm{S}]$ for more information about Lévy processes and [ØS2] for the stochastic control of jump diffusions.

\subsection{Elements of Malliavin calculus}

Since $B$ and $\widetilde{N}$ are independent we may assume that

$$
\Omega:=\Omega_{B} \times \Omega_{\widetilde{N}}, \quad \mathcal{F}:=\mathcal{F}_{B} \otimes \mathcal{F}_{\widetilde{N}}, \quad P:=P_{B} \otimes P_{\widetilde{N}}
$$

where $\left(\Omega_{i}, \mathcal{F}_{i}, P_{i}\right), i=B, \widetilde{N}$, are the probability spaces generated by $B$ and $\widetilde{N}$, respectively. Correspondingly we may consider $\mathbb{F}_{i}:=\left\{\mathcal{F}_{i, t}, t \geq 0\right\}, i=B, \widetilde{N}$, as the flow of events generated respectively be $B$ and $\widetilde{N}$ and augmented of the events of null probability $P_{i}, i=B, \widetilde{N}$, correspondingly. Then we can set $\mathcal{F}_{t}:=\mathcal{F}_{B, t} \otimes \mathcal{F}_{\widetilde{N}, t}, t \geq 0$.

From now on we fix a terminal time $T>0$ constant.

In this context we will use two types of Malliavin derivatives of an $\mathcal{F}_{T}$-measurable random variable $F=F(\omega)=F\left(\omega_{1}, \omega_{2}\right), \omega=\left(\omega_{1}, \omega_{2}\right) \in \Omega_{B} \times \Omega_{\widetilde{N}}$ :

(i) The Malliavin derivative $D_{t} F=\frac{\partial F}{\partial \omega_{1}}(t)$, with respect to $B$

(ii) The Malliavin derivative $D_{t, z} F=\frac{\partial F}{\partial \omega_{2}}(t, z)$, with respect to $\tilde{N}$.

Both derivatives are defined in terms of the respective chaos expansions (see [I]) of $F$ : 
(i) For any $\omega_{2} \in \Omega_{\widetilde{N}}$ fixed, let $F=\sum_{n=0}^{\infty} I_{n}\left(f_{n}\right)$ be the chaos expansion representation of $F\left(\cdot, \omega_{2}\right) \in L_{2}\left(P_{B}\right)$ in terms of iterated integrals $I_{n}\left(f_{n}\right)$ of $f_{n}$ with respect to $B, f_{n}$ being deterministic symmetric functions in $L_{2}\left(\lambda^{n}\right)$. If $F \in \mathbb{D}_{1,2}^{B}$, i.e.

$$
\|F\|_{\mathbb{D}_{1,2}^{B}}^{2}:=\sum_{n=1}^{\infty} n n !\left\|f_{n}\right\|_{L_{2}\left(\lambda^{n}\right)}^{2}<\infty
$$

we define

$$
D_{t} F=\sum_{n=1}^{\infty} n I_{n-1}\left(f_{n}(\cdot, t)\right), \quad t \in[0, T] .
$$

Here $\lambda$ denotes the Lebesgue measure on $[0, T]$. It is easily seen that if $F \in \mathbb{D}_{1,2}^{B}$, then

$$
\left\|D_{t} F\right\|_{L^{2}(\lambda \times P)}^{2}=\sum_{n=1}^{\infty} n n !\left\|f_{n}\right\|_{L_{2}\left(\lambda^{n}\right)}^{2}=\|F\|_{\mathbb{D}_{1,2}^{B}}^{2} .
$$

A random variable belonging to $\mathbb{D}_{1,2}^{B}$ is said to be Malliavin differentiable with respect to $B$.

(ii) For any $\omega_{1} \in \Omega_{B}$ fixed, let $F=\sum_{n=0}^{\infty} I_{n}\left(f_{n}\right)$ be the chaos expansion of $F\left(\omega_{1}, \cdot\right) \in$ $L_{2}\left(P_{\widetilde{N}}\right)$ in terms of iterated integrals $I_{n}\left(f_{n}\right)$ of $f_{n}$ with respect to $\widetilde{N}, f_{n}$ being deterministic symmetric functions in $L_{2}\left((\lambda \times \nu)^{n}\right)$. If $F \in \mathbb{D}_{1,2}^{\widetilde{N}}$, i.e.

$$
\|F\|_{\mathbb{D}_{1,2}^{\widetilde{N}}}^{2}:=\sum_{n=1}^{\infty} n n !\left\|f_{n}\right\|_{L_{2}\left((\lambda \times \nu)^{n}\right)}^{2}<\infty
$$

we define

$$
D_{t, z} F=\sum_{n=1}^{\infty} n I_{n-1}\left(f_{n}(\cdot, t, z)\right), \quad(t, z) \in[0, T] \times \mathbb{R}_{0}
$$

In this case we get that if $F \in \mathbb{D}_{1,2}^{\widetilde{N}}$, then

$$
\left\|D_{t, z} F\right\|_{L^{2}(\lambda \times \nu \times P)}^{2}=\sum_{n=1}^{\infty} n n !\left\|f_{n}\right\|_{L_{2}\left((\lambda \times \nu)^{n}\right)}^{2}=\|F\|_{\mathbb{D}_{1,2}^{\widetilde{N}}}^{2} .
$$

A random variable belonging to $\mathbb{D}_{1,2}^{\widetilde{N}}$ is said to be Malliavin differentiable with respect to $\tilde{N}$.

By considering finite sums of iterated integrals we see that $\mathbb{D}_{1,2}^{B} \cap \mathbb{D}_{1,2}^{\widetilde{N}}$ is dense in $L_{2}(P)$. In this paper we will use the following basic properties of the Malliavin derivatives: 


\section{Theorem 2.1 Chain rule I.}

(i) (Cf. $[\mathrm{N}]$ ). Let $G \in \mathbb{D}_{1,2}^{B}$ and $g \in C^{1}(\mathbb{R})$ with bounded derivative. Then $g(G) \in$ $\mathbb{D}_{1,2}^{B}$ and

$$
D_{t} g(G)=g^{\prime}(G) D_{t} G \text {. }
$$

(ii) (Cf. $[\mathrm{DM} \varnothing \mathrm{P}],[\mathrm{D} \varnothing \mathrm{P}])$. Let $G \in \mathbb{D}_{1,2}^{\widetilde{N}}$ and $g$ a real function on $\mathbb{R}$ such that $g\left(G+D_{t, z} G\right)-g(G)$ belongs to $L_{2}(\lambda \times \nu \times P)$. Then $g(G) \in \mathbb{D}_{1,2}^{\widetilde{N}}$ and

$$
D_{t, z} g(G)=g\left(G+D_{t, z} G\right)-g(G) .
$$

\section{Theorem 2.2 Duality formulae.}

(i) (Cf. $[\mathrm{N}]$ ). Let $F \in \mathbb{D}_{1,2}^{B}$ and let $\varphi(t), t \in[0, T]$, be an $\mathbb{F}$-predictable process satisfying

$$
E\left[\int_{0}^{T} \varphi^{2}(t) d t\right]<\infty
$$

Then

$$
E\left[F \cdot \int_{0}^{T} \varphi(t) d B(t)\right]=E\left[\int_{0}^{T} \varphi(t) \cdot D_{t} F d t\right]
$$

(ii) (Cf. [BL], [DMØP]). Let $F \in \mathbb{D}_{1,2}^{\widetilde{N}}$ and let $\psi(t, z), t \in[0, T], z \in \mathbb{R}_{0}$, be an $\mathbb{F}$-predictable process satisfying

$$
E\left[\int_{0}^{T} \int_{\mathbb{R}_{0}} \psi^{2}(t, z) \nu(d z) d t\right]<\infty .
$$

Then

$$
E\left[F \cdot \int_{0}^{T} \int_{\mathbb{R}_{0}} \psi(t, z) d \widetilde{N}(t, z)\right]=E\left[\int_{0}^{T} \int_{\mathbb{R}_{0}} \psi(t, z) \cdot D_{t, z} F \nu(d z) d t\right]
$$

We refer to e.g. $[\mathrm{M}],[\mathrm{N}]$ for more information about Malliavin calculus in the Brownian motion case, and to e.g. [BL], [DMØP] and the forthcoming monograph $[\mathrm{D} \varnothing \mathrm{P}]$ for the general Lévy process case. Different approaches to Malliavin calculus in the case of $\widetilde{N}$ are given in e.g. [NS] and [LSUV].

Here below we present some extension of the Malliavin derivative with respect to the Brownian motion which will be used to obtain a more general chain rule. To this aim we use $\Omega_{B}=\mathcal{S}^{\prime}(\mathbb{R})$ as the space of tempered distributions on $\mathbb{R}$.

Definition 2.3 Let $F\left(\cdot, \omega_{2}\right) \in L_{2}\left(P_{B}\right)$ be $\mathcal{F}_{T}$-measurable and let $\gamma \in L_{2}(\mathbb{R})$ (deterministic). 
(a) For any fixed $\omega_{2} \in \Omega_{\widetilde{N}}$, we say that $F$ has a directional derivative in the direction $\gamma$ if

$$
D_{\gamma} F\left(\omega_{1}, \omega_{2}\right):=\lim _{\varepsilon \rightarrow 0} \frac{F\left(\omega_{1}+\varepsilon \gamma, \omega_{2}\right)-F\left(\omega_{1}, \omega_{2}\right)}{\varepsilon}
$$

exists with convergence in probability. Then $D_{\gamma} F$ is called the directional derivative of $F$ in the direction $\gamma$.

(b) For any fixed $\omega_{2} \in \Omega_{\widetilde{N}}$, we say that $F$ is Malliavin differentiable in probability if there exists a process $\Psi(t), t \geq 0$, such that

$$
\int_{\mathbb{R}} \Psi^{2}\left(t, \omega_{1}, \omega_{2}\right) d t<\infty \quad P-\text { a.s. }
$$

and

$$
D_{\gamma} F\left(\omega_{1}, \omega_{2}\right)=\int_{\mathbb{R}} \Psi\left(t, \omega_{1}, \omega_{2}\right) \gamma(t) d t \quad \text { for all } \gamma \in L_{2}(\mathbb{R}) .
$$

If this is the case, we put $\Psi(t)=\mathcal{D}_{t} F$ and call this the Malliavin derivative in probability of $F$ with respect to $B$.

Lemma 2.4 Chain rule II. Suppose $D_{\gamma} F$ exists for some $\gamma \in L_{2}(\mathbb{R})$ and that $g \in$ $C^{1}(\mathbb{R})$. Then $g(F)$ has a directional derivative in the direction $\gamma$ and

$$
D_{\gamma} g(F)=g^{\prime}(F) D_{\gamma} F \text {. }
$$

Proof. Consider the equations

$$
\begin{aligned}
\lim _{\varepsilon \rightarrow 0} \frac{g\left(F\left(\omega_{1}+\varepsilon \gamma, \omega_{2}\right)\right)-g\left(F\left(\omega_{1}, \omega_{2}\right)\right)}{\varepsilon} & =\lim _{\varepsilon \rightarrow 0} \frac{g\left(F\left(\omega_{1}, \omega_{2}\right)+\varepsilon D_{\gamma} F\left(\omega_{1}, \omega_{2}\right)\right)-g\left(F\left(\omega_{1}, \omega_{2}\right)\right)}{\varepsilon} \\
& =\lim _{\varepsilon \rightarrow 0} \frac{g^{\prime}\left(F\left(\omega_{1}, \omega_{2}\right)\right) \varepsilon D_{\gamma} F\left(\omega_{1}, \omega_{2}\right)}{\varepsilon} \\
& =g^{\prime}\left(F\left(\omega_{1}, \omega_{2}\right)\right) D_{\gamma} F\left(\omega_{1}, \omega_{2}\right),
\end{aligned}
$$

with convergence in probability.

Theorem 2.5 Chain rule III. Suppose $F$ is Malliavin differentiable in probability. Let $g \in C^{1}(\mathbb{R})$. Then $g(F)$ is Malliavin differentiable in probability and

$$
\mathcal{D}_{t} g(F)=g^{\prime}(F) \mathcal{D}_{t} F \quad(t, \omega)-\text { a.e. }
$$

Proof. By Lemma 2.4 we have

$$
\begin{aligned}
D_{\gamma} g(F) & =g^{\prime}(F) D_{\gamma} F \\
& =g^{\prime}(F) \int_{\mathbb{R}} \gamma(t) \mathcal{D}_{t} F d t \\
& =\int_{\mathbb{R}} \gamma(t) g^{\prime}(F) \mathcal{D}_{t} F d t \quad \text { for all } \gamma \in L_{2}(\mathbb{R}),
\end{aligned}
$$

and the result follows. 
We now proceed to prove that $\mathcal{D} F$ is an extension of $D F$.

Theorem 2.6 Let $\omega_{2} \in \Omega_{\widetilde{N}}$ be fixed. Suppose $F \in \mathbb{D}_{1,2}^{B}$, then $F$ is Malliavin differentiable in probability and

$$
\mathcal{D}_{t} F=D_{t} F
$$

Proof. Let $e \in L_{2}(\mathbb{R})$ with $\int_{\mathbb{R}} e^{2}(t) d t=1$. Consider the iterated Itô integral

$$
I_{n}\left(e^{\otimes n}\right):=n ! \int_{\mathbb{R}} \int_{-\infty}^{t_{n}} \cdots \int_{-\infty}^{t_{2}} e\left(t_{1}\right) \cdots e\left(t_{n}\right) d B\left(t_{1}\right) \cdots d B\left(t_{n}\right) .
$$

By a result of Itô (cf. [I]), we have that

$$
I_{n}\left(e^{\otimes n}\right)=h_{n}\left(\int_{\mathbb{R}} e(t) d B(t)\right),
$$

where $h_{n}$ is the Hermite polynomial of order $n$. Hence, by the chain rule and a basic property of Hermite polynomials, we have

$$
\begin{aligned}
\mathcal{D}_{t}\left(I_{n}\left(e^{\otimes n}\right)\right) & =h_{n}^{\prime}\left(\int_{\mathbb{R}} e(t) d B(t)\right) e(t) \\
& =n h_{n-1}\left(\int_{\mathbb{R}} e(t) d B(t)\right) e(t) \\
& \left.=n I_{n-1}\left(e^{\otimes(n-1)}\right)\right) e(t) \\
& =D_{t}\left(I_{n}\left(e^{\otimes n}\right)\right) .
\end{aligned}
$$

It follows that $\mathcal{D}_{t}$ coincides with $D_{t}$ on the finite sums of iterated integrals and, hence, also that $\mathcal{D}_{t} F$ exists for all $F \in \mathbb{D}_{1,2}^{B}$ and

$$
\mathcal{D}_{t} F=D_{t} F \quad \text { for all } F \in \mathbb{D}_{1,2}^{B} .
$$

And the result follows.

Corollary 2.7 If $F$ is Malliavin differentiable in probability and

$$
E_{P_{B}}\left[\int_{\mathbb{R}}\left(\mathcal{D}_{t} F\left(\cdot, \omega_{2}\right)\right)^{2} d t\right]<\infty
$$

then $F \in \mathbb{D}_{1,2}^{B}$.

Proof. Let $F=\sum_{n=0}^{\infty} I_{n}\left(f_{n}\right)$ be the chaos expansion of $F\left(\cdot, \omega_{2}\right) \in L_{2}\left(P_{B}\right)$. By Theorem 2.6 we have

$$
\mathcal{D}_{t}\left(I_{n}\left(f_{n}\right)\right)=D_{t}\left(I_{n}\left(f_{n}\right)\right) \quad \text { for all } n \text {. }
$$


Hence we have

$$
\begin{aligned}
\|F\|_{\mathbb{D}_{1,2}^{B}} & =\lim _{N \rightarrow \infty} \sum_{n=1}^{N} E_{P_{B}}\left[\int_{0}^{T}\left(D_{t}\left(I_{n}\left(f_{n}\right)\right)\right)^{2} d t\right] \\
& =E_{P_{B}}\left[\int_{\mathbb{R}}\left(\mathcal{D}_{t} F\right)^{2} d t\right]<\infty .
\end{aligned}
$$

Thus $F \in \mathbb{D}_{1,2}^{B}$.

In view of this last results, in the forthcoming applications we will denote the Malliavin derivative and the Malliavin derivative in probability by the same notation $D_{t}$.

\section{An optimal portfolio problem under partial information}

We consider a market model on the complete probability space $(\Omega, \mathcal{F}, P)$ (see $(2.6)$ ) with finite time horizon $T>0$ where two investments possibilities are available:

- a risk free asset with price dynamics

$$
\left\{\begin{array}{l}
d S_{0}(t)=\rho(t) S_{0}(t) d t, \quad t \in(0, T] \\
S_{0}(0)=1
\end{array}\right.
$$

- a risky asset with price dynamics

$$
\left\{\begin{array}{l}
d S_{1}(t)=S_{1}\left(t^{-}\right)\left[\mu(t) d t+\sigma(t) d B(t)+\int_{\mathbb{R}_{0}}^{\theta}(t, z) \widetilde{N}(d t, d z)\right], \quad t \in(0, T] \\
S_{1}(0)>0
\end{array}\right.
$$

The driving noises in the model are the standard Brownian motion $B(t), t \in[0, T]$, and the compensated Poisson random measure $\widetilde{N}(d t, d z),(t, z) \in[0, T] \times \mathbb{R}_{0}$, where $\mathbb{R}_{0}:=\mathbb{R} \backslash\{0\}$. Recall that $E\left[\widetilde{N}(d t, d z)^{2}\right]=\nu(d z) d t$, where $\nu(d z), z \in \mathbb{R}_{0}$, is a $\sigma$-finite Borel measure which we assume to satisfy

$$
\int_{\mathbb{R}_{0}} z^{2} \nu(d z)<\infty
$$

- cf. (2.1). The parameters $\rho(t), \mu(t), \sigma(t)$ and $\theta(t, z), t \in[0, T], z \in \mathbb{R}_{0}$, are measurable, càglàd (i.e. left continuous paths with right sided limits) stochastic processes, adapted to the filtration $\mathbb{F}:=\left\{\mathcal{F}_{t} \subseteq \mathcal{F}, t \in[0, T]\right\}$, see (2.6). We also assume that $|\sigma(t)| \leq K_{\sigma}$ and $-1+\epsilon_{\theta} \leq \theta(t, z) \leq K_{\theta}, \nu(d z) d t$-a.e., for some $\epsilon_{\theta} \in(0,1)$ and $K_{\sigma}, K_{\theta}<\infty$, and

$$
E \int_{0}^{T}\left\{|\rho(t)|+|\mu(t)|+\sigma^{2}(t)+\int_{\mathbb{R}_{0}} \theta^{2}(t, z) \nu(d z)\right\} d t<\infty .
$$


In this section we consider a dealer who has access only to some partial information of the market events. At any time $t$ the $\sigma$-algebra $\mathcal{E}_{t} \subseteq \mathcal{F}_{t}$ represents the information at his disposal. We consider the collection of $\sigma$-algebras $\mathbb{E}:=\left\{\mathcal{E}_{t}, t \in[0, T]\right\}$ to be a filtration.

We would like to note that dealers who are subjected to delays in receiving the information can also be regarded as having partial information. For example, in case $\delta>0$ is a fixed time delay, at any time $t \geq \delta$ the actual information available would be $\mathcal{E}_{t}=\mathcal{F}_{t-\delta}$, while it would be $\mathcal{E}_{t}=\mathcal{E}_{0}$ for $t<\delta$.

In view of this lack of information, the portfolio $\pi(t)$ resulted from the dealer's decisions taken at time $t$ is an $\mathcal{E}_{t}$-measurable random variable. Here the process $\pi$ represents the fraction of wealth invested in the risky asset.

Taking the point of view of such a dealer we are interested in studying the optimization problem

$$
u(x):=\sup _{\pi \in \mathcal{A}} E\left[U\left(X_{\pi}(T)\right) \mid X_{\pi}(0)=x\right]=E\left[U\left(X_{\pi^{*}}(T)\right) \mid X_{\pi}(0)=x\right],
$$

for a given utility function

$$
U:[0, \infty) \longrightarrow[-\infty, \infty)
$$

that is an increasing, concave and lower semi-continuous function and which we assume to be continuously differentiable on $(0, \infty)$. The process $X_{\pi}(t), t \in[0, T]$, given by

$$
\begin{aligned}
& d X_{\pi}(t)=X_{\pi}\left(t^{-}\right)\{[\rho(t)+(\mu(t)-\rho(t)) \pi(t)] d t \\
& \left.+\pi(t) \sigma(t) d B(t)+\int_{\mathbb{R}_{0}} \pi(t) \theta(t, z) \tilde{N}(d t, d z)\right\},
\end{aligned}
$$

represents the value process of the admissible portfolio $\pi$. The initial capital $X_{\pi}(0)=$ $x>0$ is fixed. By the Itô formula, the unique solution of the equation above is

$$
\begin{aligned}
& X_{\pi}(t)=x \exp \left\{\int_{0}^{t}\left[\rho(s)+(\mu(s)-\rho(s)) \pi(s)-\frac{1}{2} \sigma^{2}(s) \pi^{2}(s)\right] d s\right. \\
& +\int_{0}^{t} \int_{\mathbb{R}_{0}}[\log (1+\pi(s) \theta(s, z))-\pi(s) \theta(s, z)] \nu(d z) d s \\
& \left.+\int_{0}^{t} \pi(s) \sigma(s) d B(s)+\int_{0}^{t} \int_{\mathbb{R}_{0}} \log (1+\pi(s) \theta(s, z)) \tilde{N}(d s, d z)\right\},
\end{aligned}
$$

with $\pi \in \mathcal{A}$. The set of admissible controls $\mathcal{A}$ is defined as follows.

Definition 3.1 The set $\mathcal{A}$ of admissible portfolios consists of all processes $\pi=\pi(t)$, 
$t \in[0, T]$, such that

(3.6) $\quad \pi$ is càglàd and adapted to the filtration $\mathbb{E}$; $\pi(t) \theta(t, z)>-1+\epsilon_{\pi}$ for a.a. $(t, z)$ with respect to $d t \times \nu(d z)$, for some $\epsilon_{\pi} \in(0,1)$ depending on $\pi$;

$$
E \int_{0}^{T}\left\{|\mu(s)-r(s)||\pi(s)|+\sigma^{2}(s) \pi^{2}(s)+\int_{\mathbb{R}} \pi^{2}(s) \theta^{2}(s, z) \nu(d z)\right\} d s<\infty .
$$

Our forthcoming discussion aims at giving a characterization of the locally optimal portfolios in (3.3). For given $\pi \in \mathcal{A}$ we define the stochastic process $Y_{\pi}(t), t \in[0, T]$, as

$$
\begin{aligned}
& Y_{\pi}(t):=\int_{0}^{t}\left\{\mu(s)-\rho(s)-\sigma^{2}(s) \pi(s)-\int_{\mathbb{R}_{0}} \frac{\pi(s) \theta^{2}(s, z)}{1+\pi(s) \theta(s, z)} \nu(d z)\right\} d s \\
& +\int_{0}^{t} \sigma(s) d B(s)+\int_{0}^{t} \int_{\mathbb{R}_{0}} \frac{\theta(s, z)}{1+\pi(s) \theta(s, z)} \widetilde{N}(d s, d z), \quad t \in[0, T] .
\end{aligned}
$$

Then our result can be stated as follows:

Theorem 3.2 Let $\pi \in \mathcal{A}$ be such that

(3.10) the random variable $F_{\pi}(T):=U^{\prime}\left(X_{\pi}(T)\right) X_{\pi}(T) \quad$ (here $U^{\prime}(x)=\frac{d}{d x} U(x)$ ); belongs to $\mathbb{D}_{1,2}^{B} \cap \mathbb{D}_{1,2}^{\widetilde{N}}$,

(3.11) for all $\beta \in \mathcal{A}$, with $\beta$ bounded, there exists a $\delta>0$ such that the family

$$
\left\{U^{\prime}\left(X_{\pi+y \beta}(T)\right) X_{\pi+y \beta}(T) Y_{\pi+y \beta}(T)\right\}_{y \in(-\delta, \delta)}
$$

is uniformly integrable. Suppose $\pi$ is a local maximum point for the problem (3.3), then $\pi$ satisfies the equation

$$
\begin{aligned}
& E\left[\left(\mu(s)-\rho(s)-\sigma^{2}(s) \pi(s)\right) F_{\pi}(T)+\sigma(s) D_{s}\left(F_{\pi}(T)\right) \mid \mathcal{E}_{s}\right] \\
& +E\left[\int_{\mathbb{R}_{0}} \frac{\theta(s, z) D_{s, z}\left(F_{\pi}(T)\right)-\pi(s) \theta^{2}(s, z) F_{\pi}(T)}{1+\pi(s) \theta(s, z)} \nu(d z) \mid \mathcal{E}_{s}\right]=0, \quad s \in[0, T] .
\end{aligned}
$$

Conversely, suppose that (3.12) holds and that

$$
x U^{\prime \prime}(x)+U^{\prime}(x) \leq 0, \quad x>0,
$$

then $\pi$ is a local maximum for the problem (3.3). 
Remark 3.3 (i) Note that the buy-hold-sell portfolios $\beta$, i.e.

$$
\beta(s, \omega):=\alpha(\omega) 1_{(t, t+h]}(s), \quad s \in[0, T], \omega \in \Omega,
$$

belong to $\mathcal{A}$ provided that $\alpha$ is $\mathcal{E}_{t}$-measurable and such that $|\alpha| \leq K_{\alpha}$ for some sufficiently small $K_{\alpha}>0$.

(ii) If $\pi, \beta \in \mathcal{A}$ with $\beta$ bounded, then there exists $\delta>0$ such that $\pi+y \beta \in \mathcal{A}$ for all $y \in(-\delta, \delta)$.

(iii) Condition (3.13) holds if, for example, $U(x)=\log x$ or $U(x)=\frac{1}{\gamma} x^{\gamma}(\gamma<0)$.

Proof. Let us suppose that $\pi$ gives a local maximum for the problem (3.3), in the sense that

$$
E\left[U\left(X_{\pi+y \beta}(T)\right)\right] \leq E\left[U\left(X_{\pi}(T)\right)\right]
$$

for all bounded $\beta \in \mathcal{A}$ and all $y \in(-\delta, \delta)$ where $\delta>0$ is such that $\pi+y \beta \in \mathcal{A}$ (see Remark 3.3 (ii)) and (3.11) holds. For convenience let us define

$$
g(y):=E\left[U\left(X_{\pi+y \beta}(T)\right)\right], \quad y \in(-\delta, \delta) .
$$

Since the function $g$ is locally maximal at $y=0$, we have that

$$
\begin{aligned}
0 & =\frac{d}{d y} g(y)_{\mid y=0} \\
& =E\left[U ^ { \prime } ( X _ { \pi } ( T ) ) X _ { \pi } ( T ) \left\{\int _ { 0 } ^ { T } \beta ( s ) \left[\mu(s)-\rho(s)-\sigma^{2}(s) \pi(s)\right.\right.\right. \\
& \left.-\int_{\mathbb{R}_{0}}\left\{\theta(s, z)-\frac{\theta(s, z)}{1+\pi(s) \theta(s, z)}\right\} \nu(d z)\right] d s \\
& \left.\left.+\int_{0}^{T} \beta(s) \sigma(s) d B(s)+\int_{0}^{T} \int_{\mathbb{R}_{0}} \frac{\beta(s) \theta(s, z)}{1+\pi(s) \theta(s, z)} \tilde{N}(d s, d z)\right\}\right] .
\end{aligned}
$$

Let us choose the portfolio $\beta \in \mathcal{A}$ to be of the form buy-hold-sell, i.e.

$$
\beta(s)=\alpha 1_{(t, t+h]}(s), \quad 0 \leq s \leq T,
$$

for $t \in[0, T)$ and $h>0$ such that $t+h \leq T$ - see Remark 3.3 (i). Then from the equations above we have

$$
\begin{aligned}
0= & E\left[U ^ { \prime } ( X _ { \pi } ( T ) ) X _ { \pi } ( T ) \cdot \alpha \cdot \left\{\int_{t}^{t+h}\left[\mu(s)-\rho(s)-\sigma^{2}(s) \pi(s)-\int_{\mathbb{R}_{0}} \frac{\pi(s) \theta^{2}(s, z)}{1+\pi(s) \theta(s, z)} \nu(d z)\right] d s\right.\right. \\
& \left.\left.+\int_{t}^{t+h} \sigma(s) d B(s)+\int_{t}^{t+h} \int_{\mathbb{R}_{0}} \frac{\theta(s, z)}{1+\pi(s) \theta(s, z)} \tilde{N}(d s, d z)\right\}\right]
\end{aligned}
$$

which holds for all choices of $\alpha$ in the buy-hold-sell portfolios. Define

$$
F_{\pi}(T)=U^{\prime}\left(X_{\pi}(T)\right) X_{\pi}(T), \quad \text { for } \pi \in \mathcal{A},
$$


where $U^{\prime}(x)=\frac{d}{d x} U(x)$. Then we have that

$$
E\left[F_{\pi}(T)\left(Y_{\pi}(t+h)-Y_{\pi}(t)\right) \cdot \alpha\right]=0
$$

for the process $Y_{\pi}(t), t \in[0, T]$, given in (3.9). By our assumption (3.10) and the duality formula, see Theorem 2.2 , we have:

$$
E\left[F_{\pi}(T) \int_{t}^{t+h} \alpha \sigma(s) d B(s)\right]=E\left[\int_{t}^{t+h} \alpha \sigma(s) D_{s}\left(F_{\pi}(T)\right) d s\right]
$$

and

$$
\begin{aligned}
E\left[F_{\pi}(T) \int_{t}^{t+h} \int_{\mathbb{R}_{0}}\right. & \left.\frac{\alpha \theta(s, z)}{1+\pi(s) \theta(s, z)} \tilde{N}(d s, d z)\right] \\
& =E\left[\int_{t}^{t+h} \int_{\mathbb{R}_{0}} \frac{\alpha \theta(s, z) D_{s, z}\left(F_{\pi}(T)\right)}{1+\pi(s) \theta(s, z)} \nu(d z) d s\right] .
\end{aligned}
$$

Thus, substituting these two equations into (3.14) and noting that the $\sigma$-algebra generated by all the $\alpha$ 's in the buy-hold-sell portfolios coincedes with $\mathcal{E}_{t}$, we obtain

$$
\begin{gathered}
E\left[\int _ { t } ^ { t + h } \left\{\left(\mu(s)-\rho(s)-\sigma^{2}(s) \pi(s)\right) F_{\pi}(T)-\int_{\mathbb{R}_{0}} \frac{\pi(s) \theta^{2}(s, z) F_{\pi}(T)}{1+\pi(s) \theta(s, z)} \nu(d z)+\right.\right. \\
\left.\left.\sigma(s) D_{s}\left(F_{\pi}(T)\right)+\int_{\mathbb{R}_{0}} \frac{\theta(s, z) D_{s, z}\left(F_{\pi}(T)\right)}{1+\pi(s) \theta(s, z)} \nu(d z)\right\} d s \mid \mathcal{E}_{t}\right]=0 .
\end{gathered}
$$

Since this holds for all $t, h$ such that $0 \leq t<t+h \leq T$, we conclude that

$$
\begin{aligned}
& E\left[\left(\mu(s)-\rho(s)-\sigma^{2}(s) \pi(s)\right) F_{\pi}(T)+\sigma(s) D_{s}\left(F_{\pi}(T)\right) \mid \mathcal{E}_{s}\right] \\
& +E\left[\int_{\mathbb{R}_{0}} \frac{\theta(s, z) D_{s, z}\left(F_{\pi}(T)\right)-\pi(s) \theta^{2}(s, z) F_{\pi}(T)}{1+\pi(s) \theta(s, z)} \nu(d z) \mid \mathcal{E}_{s}\right]=0, \quad s \in[0, T]
\end{aligned}
$$

- see (3.12)

On the other side the argument can be reversed as follows. If we assume that (3.12) holds, then

$$
E\left[F_{\pi}(T)\left(Y_{\pi}(t+h)-Y_{\pi}(t)\right) \mid \mathcal{E}_{t}\right]=0
$$

which is equivalent to

$$
\begin{gathered}
E\left[F_{\pi}(T) \cdot \int_{0}^{T} \beta(s)\left[\mu(s)-\rho(s)-\sigma^{2}(s) \pi(s)-\int_{\mathbb{R}_{0}}\left\{\frac{\pi(s) \theta^{2}(s, z)}{1+\pi(s) \theta(s, z)}\right\} \nu(d z)\right] d s\right. \\
\left.+\int_{0}^{T} \beta(s) \sigma(s) d B(s)+\int_{0}^{T} \int_{\mathbb{R}_{0}} \frac{\beta(s) \theta(s, z)}{1+\pi(s) \theta(s, z)} \tilde{N}(d s, d z)\right]=0
\end{gathered}
$$


for all buy-hold-sell portfolios $\beta(s)=\alpha 1_{(t, t+h]}(s), 0 \leq s \leq T$. By (3.11) the function $g(y)=E\left[U\left(X_{\pi+y \beta}(T)\right)\right], y \in(-\delta, \delta)$, satisfies

$$
g^{\prime}(0)=\frac{d}{d y} g(y)_{\mid y=0}=E\left[U^{\prime}\left(X_{\pi}(T)\right) \frac{d}{d y} X_{\pi+y \beta}(T)_{\mid y=0}\right]=0
$$

for all bounded $\beta \in \mathcal{A}$. We want to conclude that $y=0$ gives a local maximum for $g$ and hence that $\pi \in \mathcal{A}$ is locally optimal for the problem (3.3). To this end, fix

$$
\begin{aligned}
h(y):= & \log X_{\pi+y \beta}(T) \\
= & \int_{0}^{T}\left\{\rho(s)+(\mu(s)-\rho(s))(\pi(s)+y \beta(s))-\frac{1}{2} \sigma^{2}(s)(\pi(s)+y \beta(s))^{2}\right\} d s \\
& -\int_{0}^{T} \int_{\mathbb{R}_{0}}\{(\pi(s)+y \beta(s)) \theta(s, z)-\log (1+(\pi(s)+y \beta) \theta(s, z))\} \nu(d z) d s \\
& +\int_{0}^{T}(\pi(s)+y \beta(s)) \sigma(s) d B(s)+\int_{0}^{T} \int_{\mathbb{R}_{0}} \log (1+(\pi(s)+y \beta) \theta(s, z)) \widetilde{N}(d s, d z) .
\end{aligned}
$$

Then

$$
g^{\prime}(y)=E\left[U^{\prime}\left(X_{\pi+y \beta}(T)\right) \frac{d}{d y} X_{\pi+y \beta}(T)\right]
$$

and

$$
g^{\prime \prime}(y)=E\left[U^{\prime \prime}\left(X_{\pi+y \beta}(T)\right)\left(\frac{d}{d y} X_{\pi+y \beta}(T)\right)^{2}+U^{\prime}\left(X_{\pi+y \beta}(T)\right) \frac{d^{2}}{d y^{2}} X_{\pi+y \beta}(T)\right],
$$

where

$$
\frac{d^{2}}{d y^{2}} X_{\pi+y \beta}(T)=\frac{d}{d y}\left(X_{\pi+y \beta}(T) h^{\prime}(y)\right)=X_{\pi+y \beta}(T) h^{\prime \prime}(y)+X_{\pi+y \beta}(T)\left(h^{\prime}(y)\right)^{2} .
$$

Note that

$$
h^{\prime \prime}(0)=-\int_{0}^{T} \sigma^{2}(s) \beta^{2}(s) d s-\int_{0}^{T} \int_{\mathbb{R}_{0}} \frac{\beta^{2}(s) \theta^{2}(s, z)}{(1+\pi(s) \theta(s, z))^{2}} N(d s, d z) \leq 0 .
$$

Therefore, we have

$$
g^{\prime \prime}(0)=E\left[U^{\prime \prime}\left(X_{\pi}(T)\right) X_{\pi}^{2}(T)\left(h^{\prime}(0)\right)^{2}+U^{\prime}\left(X_{\pi}(T)\right) X_{\pi}(T)\left(h^{\prime \prime}(0)+\left(h^{\prime}(0)\right)^{2}\right)\right]
$$

and we see that $g^{\prime \prime}(0) \leq 0$ for all bounded $\beta \in \mathcal{A}$ if

$$
U^{\prime \prime}(x) x+U^{\prime}(x) \leq 0, \quad x \geq 0 .
$$

By this the proof is complete. 
Remark 3.4 Condition (3.10) depends on the choice of utility function. Here we give some examples:

(i) By the chain rule (see Theorem 2.1), condition (3.10) holds if $X_{\pi}(T) \in \mathbb{D}_{1,2}^{B} \cap \mathbb{D}_{1,2}^{\widetilde{N}}$ (for the optimal $\pi$ ) and, if $\sigma \neq 0$,

$$
\frac{d}{d x} U^{\prime}(x) x, \quad x \in(0, \infty),
$$

is bounded - cf. (2.11). In particular, this is the case for

$$
U(x)=\log x, \quad x>0,
$$

and

$$
U(x)=-\exp \{-\lambda x\}, \quad x>0 \quad(\lambda>0) .
$$

(ii) Suppose

$$
U(x)=\frac{1}{\gamma} x^{\gamma}, \quad x>0 \quad(\gamma \in(-\infty, 1) \backslash\{0\}) .
$$

Then by Theorem 2.5 we have

$$
D_{t} F_{\pi}(T)=D_{t}\left(X_{\pi}^{\gamma}(T)\right)=\gamma X_{\pi}^{\gamma}(T) D_{t}\left(\log X_{\pi}(T)\right),
$$

and, by Theorem 2.1 (ii), we have

$$
\begin{aligned}
D_{t, z} F_{\pi}(T) & =\left(X_{\pi}(T)+D_{t, z} X_{\pi}(T)\right)^{\gamma}-X_{\pi}^{\gamma}(T) \\
& =X_{\pi}^{\gamma}(T)\left[\exp \left\{\gamma D_{t, z} \log X_{\pi}(T)\right\}-1\right],
\end{aligned}
$$

provided that $\log X_{\pi}(T)$ is Malliavin differentiable in probability with respect to $B$ and belongs to $D_{1,2}^{\widetilde{N}}$. For this it suffices that $\rho(s), \mu(s), \sigma(s), \theta(s, z)$ and $\pi(s)$ are Malliavin differentiable for each $s$ and $(s, z)$. Note that by Corollary 2.7 we then get that $F \in \mathbb{D}_{1,2}^{B}$ if

$$
E\left[X_{\pi}^{2 \gamma}(T) \int_{0}^{T}\left(D_{t}\left(\log X_{\pi}(T)\right)\right)^{2} d t\right]<\infty
$$

Remark 3.5 Using the white noise framework, condition (3.10) on $F_{\pi}(T)$ could be relaxed and replaced by

$$
E\left[\left(F_{\pi}(T)\right)^{2}\right]<\infty .
$$

The result still holds in the same form. A proof of this is given in the forthcoming book [DØP]. 
Remark 3.6 Condition (3.11) depends on the choice of utility function and may be difficult to verify. Here we give some examples under which it holds.

(i) First consider the case in which $U^{\prime}(x) x$ is uniformly bounded for $x \in(0, \infty)$. In particular it holds for $U(x)=\log x$ and $U(x)=-\exp \{-\lambda x\}(\lambda>0)$. The condition (3.11) holds if $Y(y):=Y_{\pi+y \beta}(T), y \in(-\delta, \delta)$ is uniformly integrable. The uniform integrability of $\{Y(y)\}_{y \in(-\delta, \delta)}$ is assured by

$$
\sup _{y \in(-\delta, \delta)} E\left[|Y(y)|^{p}\right]<\infty \quad \text { for some } p>1 .
$$

Since $\pi, \beta \in \mathcal{A}$ (see (3.7)), we have that $1+(\pi(s)+y \beta(s)) \theta(s, z) \geq \epsilon_{\pi}-\delta$ $d t \times \nu(d z)$-a.e. for some $\delta$ small enough. Thus

$$
\begin{aligned}
E\left[\left(\int_{0}^{T} \int_{\mathbb{R}_{0}} \frac{\theta(s, z)}{1+(\pi(s)+y \beta(s)) \theta(s, z)} \tilde{N}(d s, d z)\right)^{2}\right] \\
\leq \frac{1}{\left(\epsilon_{\pi}-\delta\right)^{2}} E\left[\int_{0}^{T} \int_{\mathbb{R}_{0}} \theta^{2}(s, z) \nu(d z) d s\right]<\infty .
\end{aligned}
$$

Therefore we have that $E\left[Y^{2}(y)\right]$ is uniformly bounded in $y \in(-\delta, \delta)$.

(ii) In the case of power utility function

$$
U(x)=\frac{1}{\gamma} x^{\gamma}, \quad x>0 \quad \text { for some } \gamma \in(0,1),
$$

we can see that $U^{\prime}\left(X_{\pi+y \beta}(T)\right) X_{\pi+y \beta}(T)|Y(y)|=X_{\pi+y \beta}^{\gamma}(T)|Y(y)|$ and condition (3.11) would be satisfied if

$$
\sup _{y \in(-\delta, \delta)} E\left[\left(X_{\pi+y \beta}^{\gamma}(T)|Y(y)|\right)^{p}\right]<\infty \quad \text { for some } p>1 .
$$

We can write

$$
X_{\pi+y \beta}(T)=X_{\pi}(T) Z(y)
$$

where

$$
\begin{aligned}
Z(y):= & \exp \left\{\int_{0}^{T}\left[(\mu(s)-\rho(s)) y \beta(s)-\sigma^{2}(s) y \beta(s) \pi(s)-\frac{1}{2} \sigma^{2}(s) y^{2} \beta^{2}(s)\right] d s\right. \\
& +\int_{0}^{T} y \sigma(s) \beta(s) d B(s) \\
& +\int_{0}^{T} \int_{\mathbb{R}}[\log (1+(\pi(s)+y \beta(s)) \theta(s, z))-\log (1+\pi(s) \theta(s, z))-y \beta(s) \theta(s, z)] \nu(d z) d s \\
& \left.+\int_{0}^{T} \int_{\mathbb{R}}[\log (1+(\pi(s)+y \beta(s)) \theta(s, z))-\log (1+\pi(s) \theta(s, z))] \widetilde{N}(d s, d z)\right\} .
\end{aligned}
$$


From the iterated application of the Hölder inequality we have

$$
\begin{aligned}
& E\left[\left(X_{\pi+y \beta}^{\gamma}(T)|Y(y)|\right)^{p}\right] \\
& \leq\left(E\left[\left(X_{\pi}(T)\right)^{\gamma p a_{1} b_{1}}\right]\right)^{\frac{1}{a_{1} b_{1}}}\left(E\left[(Z(y))^{\gamma p a_{1} b_{2}}\right]\right)^{\frac{1}{a_{1} b_{2}}}\left(E\left[(|Y(y)|)^{p a_{2}}\right]\right)^{\frac{1}{a_{2}}},
\end{aligned}
$$

where $a_{1}, a_{2}: \frac{1}{a_{1}}+\frac{1}{a_{2}}=1$ and $b_{1}, b_{2}: \frac{1}{b_{1}}+\frac{1}{b_{2}}=1$. Then we can choose $a_{1}=\frac{2}{2-p}$, $a_{2}=\frac{2}{p}$ and also $b_{1}=\frac{2-p}{\gamma p}, b_{2}=\frac{2-p}{2-p-\gamma p}$ for some $p \in\left(1, \frac{2}{\gamma+1}\right)$. Hence

$$
\begin{aligned}
& E\left[\left(X_{\pi+y \beta}^{\gamma}(T)|Y(y)|\right)^{p}\right] \\
& \leq\left(E\left[\left(X_{\pi}(T)\right)^{2}\right]\right)^{\frac{\gamma p}{2}}\left(E\left[(Z(y))^{\frac{2 \gamma p}{2-p-\gamma p}}\right]\right)^{\frac{2-p-\gamma p}{2}}\left(E\left[(|Y(y)|)^{2}\right]\right)^{\frac{p}{2}} .
\end{aligned}
$$

If the value $X_{\pi}(T)$ in (3.5) satisfies

$$
E\left[\left(X_{\pi}(T)\right)^{2}\right]<\infty
$$

then the condition (3.11) holds if

$$
\left.\sup _{y \in(-\delta, \delta)} E\left[(Z(y))^{\frac{2 \gamma p}{2-p-\gamma p}}\right\}\right]<\infty .
$$

Condition (3.19) holds if, e.g.,

$$
E\left[\exp \left\{K\left(\int_{0}^{T}[|\mu(s)-\rho(s)|+|\pi(s)| d s)\right\}\right]<\infty \text { for all } K>0 .\right.
$$

Note that condition (3.18) is verified, for example, if for all $K>0$

$$
\begin{aligned}
& E\left[\operatorname { e x p } \left\{K \left(\int_{0}^{T}[|\mu(s)-\rho(s)|+|\pi(s)|] d s+\left|\int_{0}^{T} \pi(s) \sigma(s) d B(s)\right|\right.\right.\right. \\
& \left.\left.\left.+\left|\int_{0}^{T} \int_{\mathbb{R}} \log (1+\pi(s) \theta(s, z)) \tilde{N}(d s, d z)\right|\right)\right\}\right]<\infty .
\end{aligned}
$$

By similar arguments we can also treat the case of a utility function with $U^{\prime}(x)$ uniformly bounded for $x \in(0, \infty)$. We omit the details.

\section{Examples}

Example 4.1 Let us consider problem (3.3) with logarithmic utility, i.e.

$$
U(x)=\log x, \quad x>0 .
$$


In this case we have

$$
F_{\pi}(T)=U^{\prime}\left(X_{\pi}(T)\right) X_{\pi}(T)=1
$$

and (3.12) simplifies to

$$
\bar{\mu}(s)-\bar{\rho}(s)-\bar{\sigma}^{2}(s) \pi(s)-\pi(s) E\left[\int_{\mathbb{R}_{0}} \frac{\theta^{2}(s, z)}{1+\pi(s) \theta(s, z)} \nu(d z) \mid \mathcal{E}_{s}\right]=0, \quad s \in[0, T],
$$

where, for convenience in notation we have put $\bar{\mu}(s):=E\left[\mu(s) \mid \mathcal{E}_{s}\right], \bar{\rho}(s):=E\left[\rho(s) \mid \mathcal{E}_{s}\right]$, $\bar{\sigma}^{2}(s):=E\left[\sigma^{2}(s) \mid \mathcal{E}_{s}\right], s \in[0, T]$. This result is a synthesis of Corollary 2.13 and Theorem 3.12 in $[\varnothing \mathrm{S}]$, where the logarithmic utility case is studied in the framework of an anticipative environment. In particular,

(i) if $\theta \equiv 0$ and $\sigma>0$ in the price dynamics, then the process

$$
\pi^{*}(s)=\frac{\bar{\mu}(s)-\bar{\rho}(s)}{\bar{\sigma}^{2}(s)}, \quad s \in[0, T],
$$

belongs to $\mathcal{A}$ and is an optimal portfolio;

(ii) if the price dynamics (3.2) are driven by a Brownian motion and a centered Poisson process, i.e. $\sigma>0, \nu(d z)=\delta_{1}(d z)$ and $\theta(t, z)=z$, then equation (4.1) can be written as

$$
\bar{\mu}-\bar{\rho}-\overline{\sigma^{2}} \pi-\frac{\pi}{1+\pi} \equiv 0 .
$$

Thus the processes

$$
\pi^{*} \equiv \frac{1}{2 \bar{\sigma}^{2}}\left\{(\bar{\mu}-\bar{\rho})-\overline{\sigma^{2}}-1 \pm \sqrt{\left[(\bar{\mu}-\bar{\rho})-\bar{\sigma}^{2}-1\right]^{2}+4 \overline{\sigma^{2}}(\bar{\mu}-\bar{\rho})}\right\}
$$

are optimal for the problem (3.3) if $\pi \geq-1+\epsilon \pi$ for some $\epsilon_{\pi}>0$ (this depends on the choices of the coefficients in the price dynamics). If, in this setting, the price dynamics of the risky asset is driven by the centered Poisson process only, i.e. $\sigma \equiv 0$, then (4.1) leads to

$$
\bar{\mu}-\bar{\rho}-\frac{\pi}{1+\pi} \equiv 0
$$

Hence

$$
\pi^{*} \equiv \frac{\bar{\mu}-\bar{\rho}}{1-(\bar{\mu}-\bar{\rho})} \quad(\mu-\rho<1-\epsilon, \text { for some } \epsilon \in(0,1)),
$$

belongs to $\mathcal{A}$ and is optimal for (3.3). 
Example 4.2 Next let us consider the case with power utility, i.e.

$$
U(x)=\frac{1}{\gamma} x^{\gamma}, \quad x>0,
$$

where $\gamma \in(0,1)$ is a constant. In this case we get $F_{\pi}(T)=X_{\pi}^{\gamma}(T)$, thus, by the chain rules (see Theorem 2.1 and Theorem 2.5),

$$
D_{t} F_{\pi}(T)=\gamma X_{\pi}^{\gamma}(T) D_{t} \log X_{\pi}(T)
$$

and

$$
\begin{aligned}
D_{t, z} F_{\pi}(T) & =\left(X_{\pi}(T)+D_{t, z} X_{\pi}(T)\right)^{\gamma}-X_{\pi}^{\gamma}(T) \\
& =X_{\pi}^{\gamma}(T)\left[\exp \left\{\gamma D_{t, z} \log X_{\pi}(T)\right\}-1\right],
\end{aligned}
$$

if $\log X_{\pi}(T) \in \mathbb{D}_{1,2}^{B} \cap \mathbb{D}_{1,2}^{\widetilde{N}}$ - see Remark 3.4. Then (3.12) becomes

$$
\begin{aligned}
& E\left[X _ { \pi } ^ { \gamma } ( T ) \left(\mu(t)-\rho(t)-\sigma^{2}(t) \pi(t)\right.\right. \\
& +\int_{\mathbb{R}_{0}} \frac{\theta(t, z)}{1+\pi(t) \theta(t, z)}\left(\exp \left\{\gamma D_{t, z} \log X_{\pi}(T)\right\}-\pi(t) \theta(t, z)-1\right) \nu(d z) \\
& \left.\left.+\gamma \sigma(t) D_{t} \log X_{\pi}(T)\right) \mid \mathcal{E}_{t}\right]=0 .
\end{aligned}
$$

In particular, if the coefficients $\mu(t), \rho(t), \sigma(t)$ and $\theta(t, z), t \in[0, T], z \in \mathbb{R}_{0}$, are all deterministic and we would like to have a $\pi(t), t \in[0, T]$, deterministic also, then $\pi$ must satisfy the equation

$$
\mu(t)-\rho(t)+(\gamma-1) \sigma^{2}(t) \pi(t)+\int_{\mathbb{R}_{0}} \theta(t, z)\left[(1+\pi(t) \theta(t, z))^{\gamma-1}-1\right] \nu(d z)=0
$$

Conversely, any solution $\pi$ of the equation above is an optimal deterministic portfolio.

Remark 4.3 The main feature of equation (3.12) is that it gives an explicit relation between the optimal portfolio $\pi$ and the corresponding optimal terminal wealth $\hat{X}_{\pi}(T)=X_{\pi}(T)$. The following examples illustrate this.

Example 4.4 In the price dynamics, let us assume that $\theta=0, \sigma(t) \neq 0, t \in[0, T]$ and

$$
E\left[\exp \left\{\frac{1}{2} \int_{0}^{T}\left(\frac{\mu(s)-\rho(s)}{\sigma(s)}\right)^{2} d s\right\}\right]<\infty .
$$


Moreover we set $\mathcal{E}_{t}=\mathcal{F}_{t}$, for all $t$. In this context the market model is complete. It is known that the optimal terminal wealth $\hat{X}_{\pi}(T)$ is given by

$$
\hat{X}_{\pi}(T)=I\left(\mathcal{Y}(x) H_{0}(T)\right),
$$

where $I:=\left(U^{\prime}\right)^{-1}$ is the inverse of $U^{\prime}(u)=\frac{d}{d u} U(u)$, and $\mathcal{Y}(x)=\mathcal{X}^{-1}(x)$ is the inverse of the function $\mathcal{X}$ defined by

$$
\mathcal{X}(y)=E\left[H_{0}(T) I\left(y H_{0}(T)\right)\right],
$$

with

$$
H_{0}(T)=\exp \left\{-\int_{0}^{T} \frac{\mu(s)-\rho(s)}{\sigma(s)} d B(s)-\int_{0}^{T}\left[\rho(s)+\frac{1}{2}\left(\frac{\mu(s)-\rho(s)}{\sigma(s)}\right)^{2}\right] d s\right\}
$$

- see e.g. [KS], Chapter 3. Hence

$$
F_{\pi}(T)=U^{\prime}\left(X_{\pi}(T)\right) X_{\pi}(T)=\mathcal{Y}(x) H_{0}(T) I\left(\mathcal{Y}(x) H_{0}(T)\right)
$$

(cf. (3.10)) is known in this case and, since $H_{0}(T) \in \mathbb{D}_{1,2}^{B}$ (see assumptions on the coefficients in the price dynamics), we can solve (3.12) for $\pi$ as follows:

$$
\pi(s)=\frac{\mu(s)-\rho(s)}{\sigma^{2}(s)}+\frac{E\left[D_{s} F_{\pi}(T) \mid \mathcal{F}_{s}\right]}{\sigma(s) E\left[F_{\pi}(T) \mid \mathcal{F}_{s}\right]}, \quad s \in[0, T] .
$$

Thus, any solution $\pi$ of (4.7) is optimal for the problem (3.3). Note that if the utility function is logarithmic, then $F_{\pi}(T)=1$ and hence we find directly the classical solution to the optimization problem under full information. Cf. also Example 4.1.

Example 4.5 Here we consider an extension of Example 4.4 to the general case (3.1)(3.2) where $\sigma(t) \neq 0, \theta(t, z) \neq 0, t \in[0, T], z \in \mathbb{R}_{0}$, and hence the market is possibly incomplete. As in the previous case we assume $\mathcal{E}_{t}=\mathcal{F}_{t}$, for all $t$.

Let $u(x)$ be as in (3.3) and consider the associated dual problem

$$
v(y):=\inf _{Q \in \mathcal{M}_{a}} E\left[V\left(y \frac{d Q}{d P}\right)\right], \quad y>0,
$$

where

$$
V(\lambda):=\sup _{\xi \in \mathbb{R}}\{U(\xi)-\lambda \xi\}, \quad \lambda>0,
$$

is the Legendre transform of $U$ and $\mathcal{M}_{a}$ is the set of measures $Q$ absolutely continuous with respect to $P$. 
Then - under certain conditions - the optimal terminal wealth $\hat{X}_{\pi}(T)$ is given by

$$
\hat{X}_{\pi}(T)=I\left(y(x) \frac{d \hat{Q}(y(x))}{d P}\right)
$$

where $I:=\left(U^{\prime}\right)^{-1}$ (as in Example 4.4). Here $x>0$ is related to $y=y(x)>0$ via $u^{\prime}(x)=y$ or, equivalently, $x=-v^{\prime}(y)$, and the measure $\hat{Q}=\hat{Q}(y) \in \mathcal{M}_{a}$ is the optimal measure for the dual problem (4.8). We refer to e.g. [KSch] and the survey [Sch] and the references therein for more details. Therefore, in terms of $\hat{Q}$ we get

$$
F_{\pi}(T)=U^{\prime}\left(\hat{X}_{\pi}(T)\right) \hat{X}_{\pi}(T)=y(x) \frac{d \hat{Q}(y(x))}{d P} I\left(y(x) \frac{d \hat{Q}(y(x))}{d P}\right)
$$

- cf. (3.10). With this expression for $F_{\pi}(T)$ in hands, we can see that if $F_{\pi}(T) \in$ $\mathbb{D}_{1,2}^{B} \cap \mathbb{D}_{1,2}^{\widetilde{N}}$, then a portfolio $\pi$ is optimal if and only if it satisfies the following equation:

$$
\begin{aligned}
& \left(\mu(s)-\rho(s)-\sigma^{2}(s) \pi(s)\right) E\left[F_{\pi}(T) \mid \mathcal{F}_{s}\right]+\sigma(s) E\left[D_{s}\left(F_{\pi}(T)\right) \mid \mathcal{F}_{s}\right] \\
& +\int_{\mathbb{R}_{0}} \frac{\theta(s, z) E\left[D_{s, z}\left(F_{\pi}(T)\right) \mid \mathcal{F}_{s}\right]-\pi(s) \theta^{2}(s, z) E\left[F_{\pi}(T) \mid \mathcal{F}_{s}\right]}{1+\pi(s) \theta(s, z)} \nu(d z)=0, \quad s \in[0, T] .
\end{aligned}
$$

Acknowledgement. We would like to thank Peter Bank and Thaleia Zariphopoulou for useful discussions.

\section{References}

[A] D. Applebaum (2004): Lévy Processes and Stochastic Calculus. Cambridge University Press.

[BDLØP] F.E. Benth, G. Di Nunno, A. Løkka, B. Øksendal and F. Proske (2003): Explicit representation of the minimal variance portfolio in markets driven by Lévy processes. Math. Finance, 13, pp. 54-72.

[BL] F.E. Benth and A. Løkka (2004): Anticipative calculus for Lévy processes and stochastic differential equations. Stochastics and Stochastics Reports, 76, pp. 191211.

[DØP] G. Di Nunno, B. Øksendal and F. Proske (2008): Malliavin Calculus for Lévy Processes and Applications to Finance. Manuscript (forthcoming book, Springer).

[DMØP] G. Di Nunno, T. Meyer-Brandis, B. Øksendal and F. Proske (2005): Malliavin calculus and anticipative Itô formulae for Lévy proceses. Inf. Dim. Anal. Quantum Prob. 8, pp. 235-258. 
[FLLLT] E. Fournié, J.-M. Laszry, J. Lebouchoux, P.-L. Lions and N.Touzi (1999): Application of Malliavin calculus to Monte Carlo methods in finance. Finance and Stochastics, 3, pp. 391-412.

[KO] I.Karatzas and D. Ocone (1991): A generalized Clark representation formula, with application to optimal portfolios. Stochastics and Stochastics Reports, 34, pp. $187-220$.

[KS] I. Karatzas and S. Shreve (1998): Methods of Mathematical Finance, Springer.

[KSch] D. Kramkov and W. Schachermayer (2003): Necessary and sufficient conditions in the problem of optimal investment in incomplete markets. Ann. Appl. Probab., 13, pp. 1504-1516.

[I] K. Itô (1956): Spectral type of the shift transformation of differential processes with stationary increments. Trans. Am. Math. Spoc., 81, pp. 253-263.

[M] P. Malliavin(1997): Stochastic Analysis. Springer-Verlag.

[N] D. Nualart (2006): The Malliavin Calculus and Related Topics, Second Edition, Springer.

[NS] D. Nualart and W. Schoutens(2000): Chaotic and predictable representations for Lévy processes. Stoch. Proc. Appl., 90, pp. 109-122.

[LSUV] J.A. Léon, J.L. Solé, F. Utzet and J. Vives (2002): On Lévy processes, Malliavin calculus and market models with jumps. Finance and Stochastics, 6, pp. 197225 .

[ØS] B. Øksendal and A. Sulem (2004): Partial observation control in an anticipating environment, Russian Math. Surveys 59, pp. 161-184.

[ØS2] B. Øksendal and A. Sulem (2007) Applied Stochastic Control of Jump Diffusions. Second Edition, Springer.

[S] K.-I. Sato (1999): Lévy Processes and Infinite Divisibility. Cambridge University Press.

[Sch] W. Schachermayer (2004): Portfolio optimization in incomplete financial markets. Notes of the Scuola Normale Superiore Cattedra Galileiana, Pisa.

[YZ] J. Yong and X.Y. Zhou (1999): Stochastic Controls. Springer. 